\title{
Limited Feedback Multi-stage Beam-forming Method for Beyond-Line-of-Sight Ducting Channels
}

\author{
Ergin Dinc Ozgur B. Akan \\ Next-generation and Wireless Communications Laboratory \\ Department of Electrical and Electronics Engineering \\ Koc University, Istanbul, 34450 Turkey. \\ Email: \{edinc, akan\}@ku.edu.tr
}

\begin{abstract}
Atmospheric duct is a layer in which propagating signals are refracted back to the surface. In this way, these signals are trapped between surface and duct layer. Thus, atmospheric ducts significantly limit spreading of propagating signals, and provide lower path-loss compared to standard atmosphere even at beyond-Line-of-Sight (b-LoS) ranges. Since duct formation is highly probable in regions with high humidity such as coastal and maritime areas, atmospheric ducts can be used as a communication medium especially for naval beyond-Line-of-Sight (bLoS) links. Trapping effect of atmospheric ducts provides very narrow angle-of-arrival (AoA) span, thus large planar antenna arrays can be utilized to provide sharp electronically steerable beams. To this end, we propose a limited feedback multi-stage beam-forming method for ducting channels for the first time in the literature. At the end, we present simulation results for the spectral efficiency of ducting channels with the proposed method.
\end{abstract}

Index Terms-Atmospheric ducts, Antenna arrays, Beamforming, Propagation, Refraction

\section{INTRODUCTION}

Atmospheric ducting is an anomalous wave propagation mode in which propagating signals are ducted back to the surface. In this way, ducted signals are trapped between surface and duct layer as seen in Figure 1. Atmospheric duct can be consider as a waveguide, and trapped signals can reach beyond-Line-of-Sight (b-LoS) ranges thanks to linear spreading in the horizon [1]. Therefore, ducting channel b-LoS communication is promising especially for military communications [2] because ducting channel can provide high data rate links up to $500 \mathrm{~km}$ [3] with millisecond transmission delays and very low detect/intercept probability thanks to its narrow angular spreading [4].

Naval b-LoS communication systems mostly utilize Satellite Communications (SATCOM) which have very high transmission delays. SATCOM may also experience capacity problems in regions with low coverage. In addition, relay units can be utilized to connect b-LoS units. However, relay units may be a target for hostile attacks and set up time of relay units may waste considerable amount of mission time [4]. For these reasons, there is a strong need for a direct communication channel which can connect b-LoS units. Ducting channel b-LoS communication is a promising candidate especially in coastal and maritime regions because duct formation is highly probable in humid environments. Based on our previous analysis [3] and reviews [2], ducting channel can provide high data rate links with tens of Mbps data rate up to $500 \mathrm{~km}$ with transmission delays in the order of a few milliseconds.

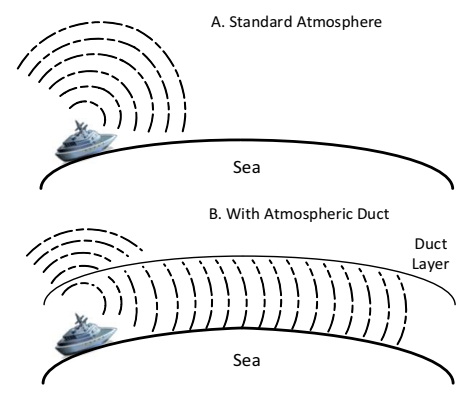

Fig. 1: Signal spreading (a) standard atmosphere, (b) atmospheric duct.

There are number of study which considers wave propagation [5]-[8] and refractivity modeling [9], [10] for atmospheric ducts. However, there are only a few paper which considers ducting channel as a communication medium. To this end, detailed review of ducting channel can be found in [2]. In [11], ducting channel is used to connect $78 \mathrm{~km}$ link with $10 \mathrm{Mbps}$ at high availability rates. We previously propose a path-loss model for surface ducts to estimate path-loss and fading characteristics of ducting channels [3]. In addition, we model angle-of-arrival (AoA) and delay spread of the channel through ray-tracing methods. In this study, we utilize the pathloss model in [3] for simulations.

Ducting channel communication has high path-losses because it is expected to operate at high ranges. Since trapped signals are concentrated in ducting layer, their AoA spread is very narrow. Therefore, antennas with sharp beam-widths can be used to provide high antenna gains. To this end, large planar antenna arrays can be utilized to provide sharp electronically steerable beams. The utilization of large antenna arrays, also known as massive MIMO, attracts significant research attention especially for LTE [12], [13] and millimeter waves [14], [15] attracts significant research attention. [14] provides Kronecker product model for planar antenna array in which azimuth and elevation beam-forming can be separated. [16] introduces virtual channel model for antenna arrays for virtual angle spreads. Since we have the knowledge of angular spreads in ducting channel through [3], we can use virtual channel 
model in [16]. In addition, [15] provides multi-resolution codebook for hierarchical beam-search. Thus, planar arrays are promising for ducting channel to detect the trapped signal by adjusting the signal beam in both elevation and azimuth direction. Although sharp angular spread of ducting channel can be exploited via large antenna arrays, there is no work which considers beam-forming for b-LoS ducting channels.

The main contributions of this paper are two-fold. Firstly, we propose a multi-stage limited feedback beam-forming method for ducting channel with planar antenna arrays. We decompose the beam-forming problem to azimuth and elevation beam-forming, and beam-forming vectors for azimuth and elevation are determined in two stage by using multi-resolution codebooks. In addition, we provide simulation results for the spectral efficiency of ducting b-LoS links for surface ducts by using the channel model developed in [3]. Although the proposed method is only tested for surface ducts, trapping characteristics of atmospheric ducts are similar, thus the proposed method can be used in all type of ducts.

The remainder of the paper is organized as follows. Section II includes the properties of ducting channel and effective trapping beam-width. Section III provides the system model for ducting channel with planar antenna arrays. Multi-stage beam-forming method is proposed in Section IV. In Section $\mathrm{V}$, simulation results are presented. Lastly, conclusions are discussed in Section VI.

Notation: We use the following notation throughout this paper: $a$ is a scalar; $\mathbf{a}$ is a column vector; $\mathbf{A}$ is a matrix and $\mathbf{a}_{k}$ is the $\mathrm{k}^{\text {th }}$ column of $\mathbf{A} ;\|\mathbf{A}\|_{F}$ is the Frobenius norm of $\mathbf{A} ;|\mathbf{A}|$ is the determinant of $\mathbf{A} ; \mathbf{A}^{T}$ is the transpose of $\mathbf{A} ; \mathbf{A}^{H}$ is the conjugate transpose of $\mathbf{A} ; \otimes$ is the Kronecker product.

\section{Ducting Channel}

In this section, we provide a brief introduction to ducting channel b-LoS communication and modeling. In addition, we explain the requirement for planar antenna arrays to provide high data rate naval b-LoS links.

\section{A. Ducting Channel Modeling}

Ducting channel wave propagation are strongly affected by complex refractivity conditions. Therefore, parabolic equation (PE) techniques are utilized to model ducting channels with their capability of modeling complex boundary conditions and refractivity of the lower atmosphere [2], [6], [7]. In addition to PE methods, ray-optics methods are also utilized in hybrid methods [8] to estimate the AoA and delay spreads [17].

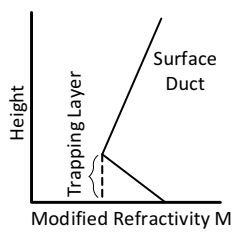

Fig. 2: Modified refractivity profile of a surface duct.

We previously introduced a channel model for surface ducts to estimate path-loss, AoA, angle-of-departure (AOD) and delay spreads [3]. Atmospheric ducts are divided into four types according to their physical formation process: evaporation duct, surface-based ducts, elevation ducts and surface ducts. Surface ducts can be modeled with a bi-linear curve as in Figure 3. We utilize PETOOL [18], which is a PE based wave propagation software, to develop the path-loss model.

In addition, we develop a ray-tracing method to estimate the ray trajectories as in Figure 3 to estimate delay spreads, AoAs, and AoDs. Based on our ray-tracing simulations in [3], ducting channel can be modeled as a narrow-band fading channel because maximum delay spread of the channel is considerably low compared to bandwidth values of interest $10-20 \mathrm{MHz} .40$ surface duct with $10 \mathrm{M}$-units duct strength has $200 \mathrm{MHz}$ one over maximum delay spread value at $400 \mathrm{~km}$. In ducting channels, coherence bandwidth decreases with increasing range and duct height because path length difference increases with these factors. Since surface ducts are mostly between $5-30 \mathrm{~m}$ heights [9], [10], ducting channel experiences narrow-band fading.

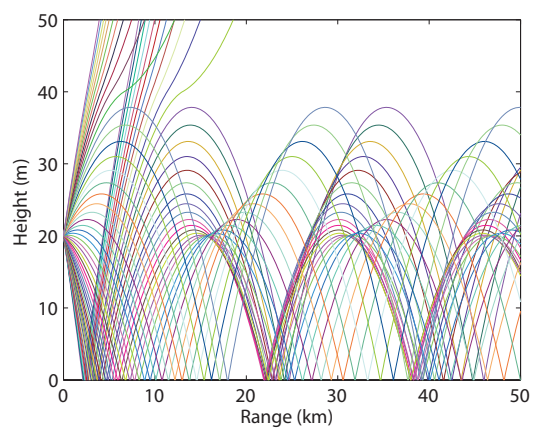

Fig. 3: Ray trajectories for $40 \mathrm{~m}$ surface duct.

\section{B. Effective Trapping Beam-width}

Trapping effect of atmospheric ducts is only effective for the rays having certain angle range depending on channel condition: transmitter height, antenna orientation; atmospheric conditions: duct height and duct strength. Rays outside of this particular range cannot be trapped and propagate through the atmosphere. Since we can only exploit the path-loss enhancements of atmospheric ducts in this angle range, antenna beamwidths and orientation should be well adjusted.

In [3], we utilize the proposed ray trajectory formula to derive the span of effective trapping beam-width which can be represented as

$$
\theta_{\text {max }, \min }^{T}= \pm \sqrt{2\left(\frac{1}{n(0)} \frac{d n}{d z}-\frac{1}{R_{0}}\right)\left(h_{t}-\delta\right)},
$$

where refractive index derivative with respect to height $d n / d z$, $h_{t}$ is the transmitter height, $\delta$ is the ducts height, $R_{0}$ is the earth radius $(\approx 6370 \mathrm{~km})$ and $n(0)$ is the index of refraction at surface $(\approx 1.00035)$ [2]. In this derivation, we assume that transmitter is in duct layer $\left(h_{t}<\delta\right)$ because this is required condition to provide trapping effect. Since $d n / d z$ is negative, 


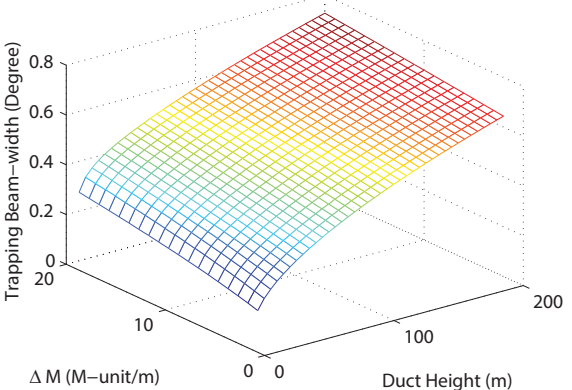

Fig. 4: Trapping beam-width values for $5 \mathrm{~m}$ antenna height.

we have real roots in (1). Instead of $d n / d z$, atmospheric ducts are characterized with duct strength $(\Delta M)$ which is the change in the modified refractivity from bottom to top of the duct $\left(d n / d z=-10^{-6}(\Delta M / \delta-0.157)\right)$ [2].

Figure 4 shows effective trapping beam-width values $\left(\theta_{\max }\right)$ for $5 \mathrm{~m}$ transmitter height for different duct heights and duct strengths. Based on experimental observations, duct heights of $10-20 \mathrm{~m}$ and duct strengths of $4-6 \mathrm{M}$-units are highly probable [9], [10]. As in Figure 4, duct height of $10-20 \mathrm{~m}$ and duct strength of $6 \mathrm{M}$-units should have $3 \mathrm{~dB}$ elevation beam-width $\left(\Psi_{e l}\right)$ of $\approx 0.25^{\circ}$. Therefore, transmitter elevation beam-width can be adjusted to this effective value to increase the utilization of the trapping effect. Receiver can focus its beam to this angle range to receive trapped rays and increase antenna gain. Therefore, we propose utilization of large planar antenna arrays to control both azimuth and elevation beams of the antenna. Since ducting channel requires very narrow elevation beams, high number of vertical row can be used to get sharper beams with high array gains.

\section{SYSTEM MODEL}

In this section, we introduce system model for ducting channel with antenna arrays. As in Figure 5, both transmitter and receiver sides have planar arrays with $W_{T} \times H_{T}=N_{T}$ antenna elements where $W_{T}$ is the number of horizontal elements, $H_{T}$ is the number of vertical elements in transmitter. Similarly, receiver also has $W_{R} \times H_{R}=N_{R}$ antenna array.

As discussed in Section II-A, ducting channel communication has narrow-band fading [3]. Since propagating signals have low spreading due to trapping effect, AoA in both elevation and azimuth is significantly narrow. Therefore, ducting channel can be modeled with the virtual channel representation as introduced in [16]. With the virtual channel representation, channel gain matrix is represented as

$$
\mathbf{H}=\sqrt{\frac{N_{T} N_{R}}{L}} \sum_{i=1}^{L} \alpha_{l} \mathbf{a}_{r}^{l}\left(\theta_{r}, \phi_{r}\right) \mathbf{a}_{t}^{l}\left(\theta_{t}, \phi_{t}\right)^{H}
$$

where $N_{T}$ is the number of transmitter, $N_{R}$ is the number of receiver, $L$ is the number of paths, $\alpha_{l}$ is the complex gain of l'th path. Since ducting channel has Rayleigh fading [3], amplitude of $\alpha_{l}$ is Rayleigh distributed. $\mathbf{a}_{t, r}$ are the array response vectors for transmitter/receiver arrays where $\theta$ is the azimuth angle and $\phi$ is the elevation of the signals. General expression for planar array response vector can be written as

$$
\begin{aligned}
\mathbf{a}(\theta, \phi)= & \frac{1}{\sqrt{N}}\left[1, \ldots, e^{j k d(m \cos (\phi) \sin (\theta)+n \sin (\phi))},\right. \\
& \left.\ldots, e^{j k d((W-1) \cos (\phi) \sin (\theta)+(H-1) \sin (\phi))}\right]^{T},
\end{aligned}
$$

where $N$ is the total number of elements in the plannar array, $W$ is the horizontal and $H$ is the vertical element number $(W \times H=N), k$ is the wavenumber $(k=2 \pi / \lambda) . d$ is the separation between array elements which is used as $\lambda / 2$ in this paper. (3) can be decomposed as

$$
\begin{aligned}
\mathbf{a}^{a z}(\theta, \phi)= & {\left[1, \ldots, e^{j k d(m \cos (\phi) \sin (\theta))},\right.} \\
& \left.\ldots, e^{j k d(W-1) \cos (\phi) \sin (\theta)}\right]^{T}, \\
\mathbf{a}^{e l}(\phi)= & {\left[1, \ldots, e^{j k d(n \sin (\phi))},\right.} \\
& \left.\ldots, e^{j k d(H-1) \sin (\phi)}\right]^{T} \\
\mathbf{a}(\theta, \phi)= & \frac{1}{\sqrt{N}}\left(\mathbf{a}^{a z}(\theta, \phi) \otimes \mathbf{a}^{e l}(\theta, \phi)\right)
\end{aligned}
$$

As noticed, $\mathbf{a}_{e l}$ only depends on elevation angle. Although, $\mathbf{a}_{a z}$ depends on both azimuth and elevation angle, elevation angle span in the ducting channel is between $-0.5^{\circ}-0.5^{\circ}$ with respect surface even at extreme cases as shown in Figure 4. Therefore, narrow elevation span of ducting channel can be used to adjust azimuth and elevation beams separately as will be discussed in Section IV.

At the end, channel gain matrix can be simplified as

$$
\begin{array}{r}
\mathbf{H}=\sqrt{\frac{1}{L} \sum_{i=1}^{L}} \alpha_{l}\left(\mathbf{a}_{r}^{a z}\left(\theta_{r}, \phi_{r}\right) \otimes \mathbf{a}_{r}^{e l}\left(\phi_{r}\right)\right) \\
\times\left(\mathbf{a}_{t}^{a z}\left(\theta_{t}, \phi_{t} \otimes \mathbf{a}_{t}^{e l}\left(\phi_{t}\right)\right)^{H}\right.
\end{array}
$$

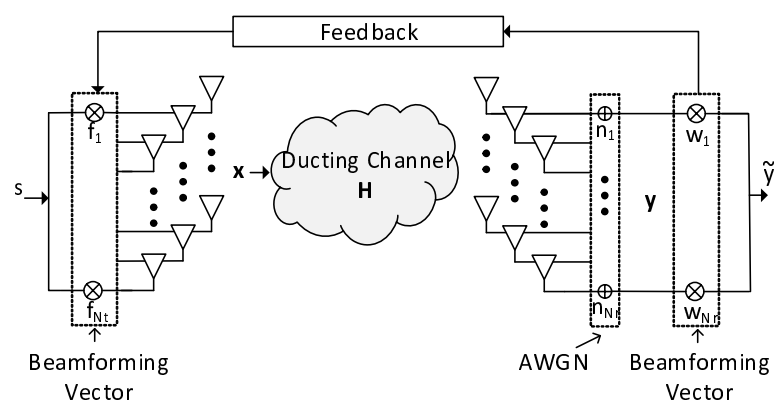

Fig. 5: Ducting channel beam-forming system model.

In Figure 5, block diagram of ducting channel system with planar array is presented. Transmitted signal can be represented as

$$
\mathbf{x}=\mathbf{f} s,
$$

where $s$ is the transmitted symbol, $\mathbf{f}_{N_{T} \times 1}$ is the transmitter beam-forming vector. Received signal can be given as

$$
\mathbf{y}=\sqrt{\rho} \mathbf{H} \mathbf{f} s+\mathbf{n},
$$


where $\mathbf{y}_{N_{R} \times 1}$ is the received signal, $\mathbf{n}_{N_{R} \times 1}$ is the noise vector. $\rho=P L \times P_{T}$ is the average received power, where $P_{T}$ is the transmit power and $P L$ is the path-loss which is directly calculated with Equation (22) in [3]. Output of the receiver planar array becomes

$$
\tilde{y}=\sqrt{\rho} \mathbf{w}^{H} \mathbf{H f} s+\mathbf{w}^{H} \mathbf{n},
$$

where $\mathbf{w}_{N_{R} \times 1}$ is the receiver beam-forming vector.

We consider single-user to single-user link. However, the proposed technique can be also extended for multiple user case by precoding matrices [19]. Therefore, spectral efficiency for single-user to single-user link can be found as

$$
R=\log _{2}\left(\left|1+\rho /\left(N_{T} \sigma_{n}^{2}\right) \mathbf{w}^{H} \mathbf{H} \mathbf{f f}{ }^{H} \mathbf{H}^{H} \mathbf{w}\right|\right)
$$

where $\sigma_{n}^{2}$ is the noise power which is modeled as thermal noise. (11) is a well-known equation and optimal beamforming vectors are found via singular value decomposition. For $\mathbf{H}=\mathbf{U} \Lambda \mathbf{V}^{H}$, optimal beam-forming vectors are $\mathbf{f}_{o p t}=\mathbf{v}_{1}$ and $\mathbf{w}_{\text {opt }}=\mathbf{u}_{1}$. However, full CSI is required at both end to achieve the optimal performance. Considering the high pathlosses and required high transmission powers for military bLoS communication, transmission of full CSI is not possible in real world conditions. Thus, we propose a limited feedback multistage beam-forming algorithm for ducting channels.

\section{Multistage Beam-Forming Algorithm}

Elevation angle span of ducting channel is very narrow as seen in Figure 4. Therefore, both receiver and transmitter is required to point their beams horizontal to the surface with very narrow beam-widths. In this way, transmitter avoids wasting the transmitting power by maximizing the amount of trapped signal energy. For $20 \mathrm{~m}$ surface duct with $6 \mathrm{M}$ units duct strength, trapping angle range in the elevation cut becomes $-0.25^{\circ}-0.25^{\circ}$. To focus the antenna beam-width to this range, 200 vertical antenna columns is required. The number of antenna elements in the azimuth cut is determined to provide desired antenna gains because the channel model in [3] is 2D. As an example, the response of $W=20 \times H=200$ planar array is presented in Figure 6 by using phased array toolbox in MATLAB. As noticed, 200 vertical elements can achieve $0.25^{\circ}$ beam-width $\left(\Psi_{e l}\right)$. Since directivity is not so sharp in azimuth cut, less number of antennas can be placed in horizontal axis to reduce the system complexity. However, it is not possible to transmit CSI for such a large antenna array, thus we propose a limited feedback beam-forming algorithm.

(4) depends on both azimuth and elevation angle, and (5) depends on only elevation angle. Although (4) is dependent to both angles, we can separate azimuth and elevation beamforming thanks to sharp elevation range. Consider planar arrays on $\mathrm{yz}$ axis and antennas are separated in $\mathrm{x}$ axis. For this link, $\phi$ in (4) and (5) becomes zero. However, waves in oceans or sea can cause changes in this angle and significantly affect the link quality. To avoid this problem, system will utilize only some portion of vertical elements when scanning azimuth direction to detect desired unit. In this way, elevation

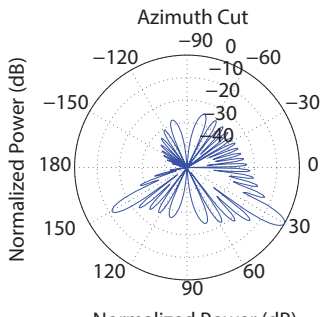

(a)

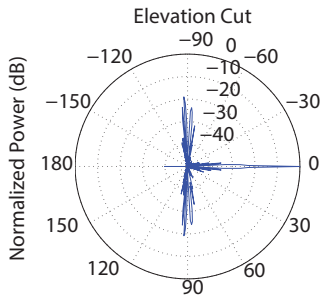

Normalized Power (dB)

(b)
Fig. 6: Planar array response for (a) azimuth cut $\left(\Psi_{e z}=\right.$ $\left.2.52^{\circ}\right)$, and (b) elevation cut $\left(\Psi_{e l}=0.25^{\circ}\right)$.

beam-width of the system will be considerably increase as shown in Figure 7 for $20 \times 10$ planar antenna array. With the increasing beam-width, receiver can detect the transmitter in wavy oceans. The number of active vertical elements can be adjusted according to operation conditions. After finding azimuth vector, all of the vertical elements are start to operate to focus the beam in elevation cut to desired direction and increase array gain.

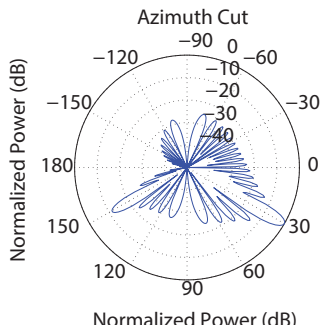

(a)

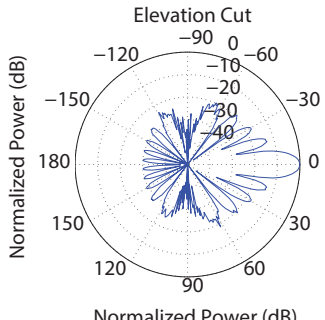

(b)
Fig. 7: Planar array response for (a) azimuth cut $\left(\Psi_{a z}=\right.$ $\left.2.52^{\circ}\right)$, and (b) elevation cut $\left(\Psi_{e l}=5.12^{\circ}\right)$.

In [14], 3D correlation matrix is decomposed as the Kronecker product of azimuth and elevation correlations. That is, azimuth and elevation beam-forming can be performed separately, and still similar eigenvalues can be maintained. Therefore, beam-forming vectors can be decomposed as

$$
\mathbf{f}_{e s t}=\mathbf{f}^{a z} \otimes \mathbf{f}^{e l}, \mathbf{w}_{e s t}=\mathbf{w}^{a z} \otimes \mathbf{w}^{e l}
$$

where $(\mathbf{f}, \mathbf{w})_{\text {est }}$ are the estimated transmit/receive beamforming vectors with the proposed method. Azimuth and elevation beam-forming vectors is determined in two stage.

\section{A. Azimuth Beam-forming Stage}

In azimuth beam-forming stage, $H_{S}$ number of vertical elements are used to get higher elevation beam-width. Therefore, elevation beam-forming vectors for azimuth scanning stage can be formulated as

$$
\begin{aligned}
\mathbf{f}_{S}^{e l} & =\left[1, e^{j k d\left(n \sin \left(\phi_{T}\right)\right)}, \ldots, e^{j k d\left(H_{S}-1\right) \sin \left(\phi_{T}\right)}, 0, \ldots, 0\right]^{T} \\
\mathbf{w}_{S}^{e l} & =\left[1, e^{j k d\left(n \sin \left(\phi_{R}\right)\right)}, \ldots, e^{j k d\left(H_{S}-1\right) \sin \left(\phi_{R}\right)}, 0, \ldots, 0\right]^{T}
\end{aligned}
$$

where $\phi_{T, R}$ are the elevation angles which makes the beam horizontal to surface. In the simulations, we assume that planar 
arrays are placed on yz axis, thus $\phi_{T, R}=0$. However, planar array can have any orientation or changing orientation because it is adaptively changed during second stage.

We assume that receiver has perfect CSI. Therefore, azimuth beam-forming vectors can be found by

$$
\left\{\mathbf{f}_{e s t}^{a z}, \mathbf{w}_{e s t}^{a z}\right\}=\underset{\forall \mathbf{w}_{k} \in \mathcal{W}, \forall \mathbf{f}_{k} \in \mathcal{F}}{\arg \max }\left\|\left(\mathbf{w}_{k} \otimes \mathbf{w}_{S}^{e l}\right)^{H} \mathbf{H}\left(\mathbf{f}_{k} \otimes \mathbf{f}_{S}^{e l}\right)\right\|
$$

where $\mathcal{F}$ and $\mathcal{W}$ are the azimuth codebooks for transmitter and receiver.

For codebook design, we utilize multi-resolution codebook approach used in [15] to provide faster search. In this approach, AoD range is divided into hierarchical sub-levels $\left(\{2 \pi u / N\}_{u \in \mathcal{I}_{a z}(s, k, m)}\right)$. Consider $S$ level codebook and $s=$ $1, \ldots, s, \ldots, S$ represent the codebook levels. In each level, there are $K^{(s-1)}$ subsets as shown in Figure 8(a). In each level, there are $K$ number of beam-forming vector $(m=1, \ldots, k, \ldots, K)$ and represented as [15]

$$
\left(\mathbf{f}_{s, k, m}^{a z}\right)^{H} \mathbf{a}_{t}^{a z}\left(\theta_{u}\right)= \begin{cases}C_{s} & \text { if } u \in \mathcal{I}_{a z}(s, k, m) \\ 0 & \text { otherwise }\end{cases}
$$

where $C_{s}$ is used for normalization and,

$$
\begin{array}{r}
\mathcal{I}_{a z}(s, k, m)=\left\{N / K^{s}(K(k-1)+m-1)+1, \ldots,\right. \\
\left.N / K^{s}(K(k-1)+m)+1\right\} .
\end{array}
$$

Let $\mathbf{A}_{T}^{a z}=\left[\mathbf{a}^{a z}\left(\theta_{1}\right), \mathbf{a}^{a z}\left(\theta_{2}\right), \ldots, \mathbf{a}^{a z}\left(\theta_{N}\right)\right]$, the beam-forming vectors can be designed as

$$
\left(\mathbf{A}_{T}^{a z}\right)^{H} \mathbf{f}_{(s, k)}=C_{s} \mathbf{g}_{(s, k)}
$$

where $\mathbf{g}_{(s, k)}$ is a $N \times 1$ column vector which has 1 's for $u \in \mathcal{I}_{a z}(s, k, m)$ and 0 's for $u \notin \mathcal{I}_{a z}(s, k, m)$. Therefore, solution to (17) can be approximated as $\mathbf{f}_{(s, k)}=$ $\left(\mathbf{A}_{T}^{a z}\left(\mathbf{A}_{T}^{a z}\right)^{H}\right)\left(\mathbf{A}_{T}^{a z}\right) \mathbf{g}_{(s, k)}$. The codebook design for the receiver is the same. In the codebook search in (14), algorithm tries to find a lower level maximizing vector by throwing lower portions. When sub-levels are smaller than the upper level, the algorithm settles to the solution. At the end, the estimated transmitter azimuth beam-forming vector is sent back to transmitter.

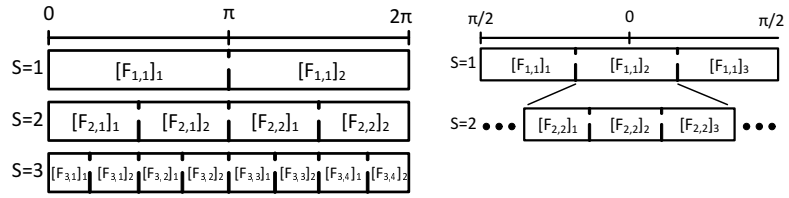

Fig. 8: (a) Multi-resolution azimuth codebook for $N=8$ and $K=2$, (b) Elevation codebook for $N=9$ and $K=3$.

\section{B. Elevation Beam-forming Stage}

After azimuth beam-forming vectors are determined, unused elements in the vertical elements starts to operate to focus the antenna beams to the effective trapping range in elevation. Elevation beam-forming vectors are determined by

$$
\left\{\mathbf{f}_{\text {est }}^{e l}, \mathbf{w}_{\text {est }}^{e l}\right\}=\underset{\forall \mathbf{w}_{k}^{e l} \in \mathcal{K}, \mathbf{f}_{k}^{e l} \in \mathcal{L},}{\arg \max }\left\|\left(\mathbf{w}_{\text {est }}^{a z} \otimes \mathbf{w}_{k}^{e l}\right)^{H} \mathbf{H}\left(\mathbf{f}_{\text {est }}^{a z} \otimes \mathbf{f}_{k}^{e l}\right)\right\| .
$$

where $\mathcal{K}$ and $\mathcal{L}$ are the elevation codebooks for transmitter and receiver.

Since we assume that planar array is on yz axis, elevation beam is required to be centered at 0 . For this reason, the major difference in elevation codebook design is the number of beam-forming vectors in each stage $K$. If $K$ takes a even value, we will not have any code which centers at 0 as in Figure 8(a). However, beam codes can be centered on 0 for an odd $K$ as in Figure 8(b). For sub-level angle range $\left(\{\pi v / N-\pi / 2\}_{v \in \mathcal{I}_{e l}(s, k, m)}\right)$, elevation codebook can be found with

$$
\left(\mathbf{f}_{s, k, m}^{e l}\right)^{H} \mathbf{a}_{t}^{e l}\left(\phi_{v}\right)= \begin{cases}1 & \text { if } v \in \mathcal{I}_{e l}(s, k, m) \\ 0 & \text { otherwise }\end{cases}
$$

For the elevation codebook, the same methodology is utilized as in azimuth case. At the end, elevation beam-forming vector is also fed back to transmitter and spectral efficiency for the proposed method can be calculated by (11) and (12).

\section{Simulation Results}

In this section, we provide simulation results for the proposed method by using the ducting channel model [3]. The simulations are performed in MATLAB. 1000 realizations are used for path-loss and 1000 realizations are utilized for channel gain matrix. Figure 9 shows the path-loss for different duct heights for $5 \mathrm{~m}$ transmitter and receiver, $10 \mathrm{M}$-units duct strength, and $10.5 \mathrm{GHz}$ frequency. Lower duct heights have lower path-loss because signal spreading increases with increasing duct height. We utilize $N=256$ and $K=2$ azimuth codebook, and $N=729$ and $K=9$ elevation codebook to support lowest of $0.25^{\circ}$ elevation beam-width The codebook size can be adaptively changed according to operation environment. $20 \times 200$ planar antenna arrays are used at both sides, and only 20 vertical rows are used in the azimuth beam-forming stage.

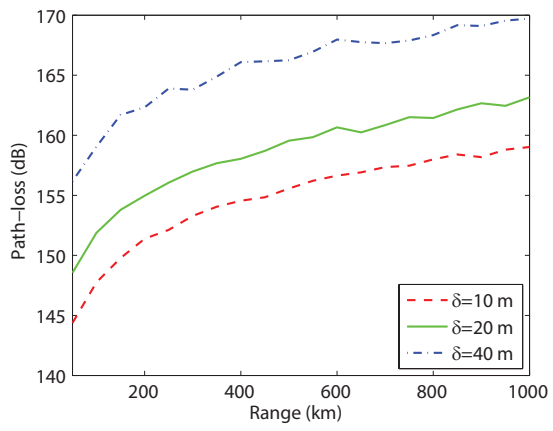

Fig. 9: Ducting channel path-loss ( $\Delta M=10$ M-units)

Figure 10(a) shows the simulation results for spectral efficiency of the ducting channel with the following channel parameters: $1 \mathrm{~W}$ transmit power, $5 \mathrm{~m}$ transmitter and receiver, 10 M-units duct strength, $10.5 \mathrm{GHz}$ frequency, number of paths $L=10$, bandwidth $10 \mathrm{MHz}$, noise temperature 300 $\mathrm{K}\left(\sigma_{n}^{2}=k T B\right.$ where $k$ is the Boltzman constant, $T$ is the temperature and $B$ is the bandwidth), $200 \mathrm{~km}$ range. As 


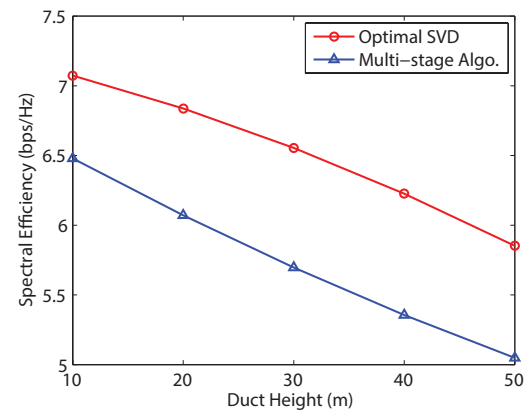

(a)

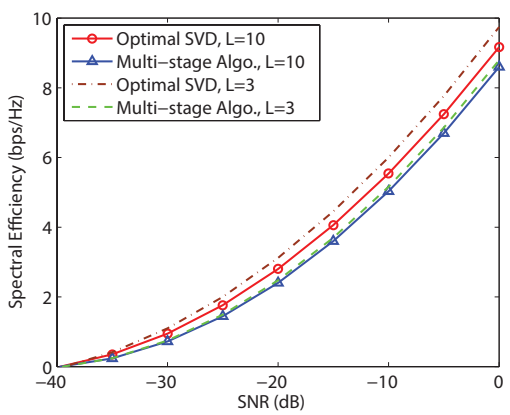

(b)

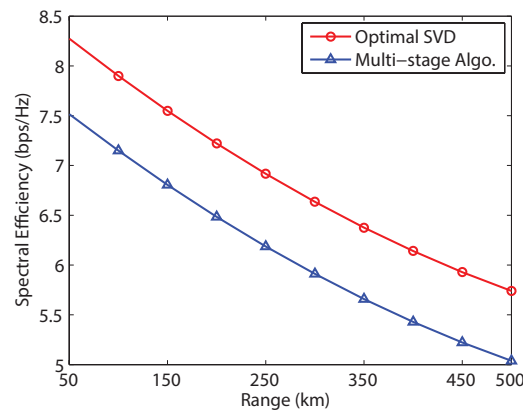

(c)

Fig. 10: (a) Spectral efficiency vs. duct height $(L=10)$, (b) Spectral efficiency vs. SNR (10 m duct height), (c) Spectral efficiency vs. range ( $L=10,10 \mathrm{~m}$ duct height).

expected, the spectral efficiency decreases with duct height because signal spreading increases as the duct height increase. Figure 10(b) shows spectral efficiency vs. SNR for $200 \mathrm{~km} \mathrm{link}$ with $10 \mathrm{~m}$ duct height. As noticed, the proposed multi-stage beam-forming method is close to the optimal case for different number of received paths. As the number of paths increase, the performance gets lower because the beam-forming vector becomes wider. Lastly, Figure 10(c) presents spectral efficiency vs. range for $10 \mathrm{~m}$ duct height and $L=10$. As expected, spectral efficiency decreases with range. However, the link can maintain $5 \mathrm{~b} / \mathrm{Hz}$ spectral efficiency at $500 \mathrm{~km}$. Therefore, we can conclude that ducting channel can be utilized in high data rate b-LoS applications. In addition, the proposed limited feedback method has close performance to optimal case as seen in Figure 10(b).

\section{CONCLUSION}

In this paper, we propose a limited feedback multi-stage beam-forming method for b-LoS ducting channel for the first time in the literature. Based on our simulations, our proposed method provides $5-8 \%$ less performance compared to optimal conditions. Since it is not possible to maintain full CSI in a communication channel with high path-loss and very large antenna array, our method is promising for ducting channel b-LoS communications thanks to the limited feedback requirement. In addition, $b-\operatorname{LoS}$ ducting channel can achieve $5 \mathrm{~b} / \mathrm{Hz}$ spectral efficiency even at $500 \mathrm{~km}$. Therefore, high data rate naval b-LoS communications can be provided with atmospheric ducts with millisecond transmission delays.

\section{REFERENCES}

[1] H. V. Hitney, J. H. Richter, R. A. Pappert, K. D. Anderson, G. B. Jr Baumgartner, "Tropospheric radio propagation assessment," Proceedings of the IEEE, vol. 73, no. 2, pp. 265-283, Feb. 1985.

[2] E. Dinc, O. B. Akan, "Beyond-Line-of-Sight Communications with Ducting Layer," IEEE Communications Magazine, vol. 52, no. 10, pp. 37-43, October 2014.

[3] E. Dinc, O. B. Akan, "Channel Model for the Surface Ducts: Large-scale Path-Loss, Delay Spread, and AOA," to appear in IEEE Transactions on Antennas and Propagation, 2015.

[4] M. J. Luddy, J. H. Winters, A. Lackpour. (2011). Beyond Lineof-Sight Communications with Smart Antennas (BLoSSA) [online]. Available:http://www.atl.1mco.com/papers/1978.pdf [Accessed on 27 Apr. 2015].
[5] V. A. Fock, "Solution of the problem of propagation of electromagnetic waves along the earths surface by method of parabolic equations," J. Phys. USSR, vol. 10, pp. 1335, 1946.

[6] M. Levy, Parabolic Equation Methods for Electromagnetic Wave Propagation. London, U.K., Institution of Electrical Engineers, 2000.

[7] I. Sirkova, "Brief review on PE method application to propagation channel modeling in sea environment" Central European Journal of Engineering, vol. 2, issue 1, pp. 19-38, 2012.

[8] Users Manual for Advanced Refractive Effects Prediction System (AREPS) . San Diego, CA: Space and Naval Warfare Systems Center, pp. 17, 2004.

[9] S. S. Mentes, Z. Kaymaz, "Investigation of Surface Duct Conditions over Istanbul, Turkey," Journal of Applied Meteorology and Climatology, vol. 46, pp 318-338, 2007.

[10] S. M. Babin "Surface Duct Height Distributions for Wallops Island, Virginia, 1985-1994," J. Appl. Meteor., vol. 35, pp. 8693, 1996.

[11] G. S. Woods, A. Ruxton, C. Huddlestone-Holmes, G. Gigan, "HighCapacity, Long-Range, Over Ocean Microwave Link Using the Evaporation Duct," IEEE Journal of Oceanic Engineering, vol. 34, no. 3, pp. 323-330, July 2009.

[12] J. Mietzner, R. Schober, L. Lampe, W. H. Gerstacker, P. A. Hoeher, "Multiple-antenna techniques for wireless communications - a comprehensive literature survey," IEEE Communications Surveys \& Tutorials, vol. 11, no. 2, pp. 87-105, Second Quarter 2009.

[13] E. Larsson, O. Edfors, F. Tufvesson, T. Marzetta, "Massive MIMO for next generation wireless systems," IEEE Communications Magazine, vol. 52, no. 2, pp. 186-195, Feb. 2014.

[14] D. Ying, F. W. Vook, T. A. Thomas, D. J. Love, A. Ghosh, "Kronecker product correlation model and limited feedback codebook design in a 3D channel model," Proceeding of ICC, pp. 5865-5870, 10-14 June 2014.

[15] A. Alkhateeb, O. El Ayach, G. Leus, R. W. Heath, "Channel Estimation and Hybrid Precoding for Millimeter Wave Cellular Systems," IEEE Journal of Selected Topics in Signal Processing, vol. 8, no. 5, pp. 831846, Oct. 2014.

[16] A. M. Sayeed, "Deconstructing multiantenna fading channels," IEEE Trans. Signal Processing, vol. 50, no. 10, pp. 2563-2579, Oct 2002.

[17] R. Akbarpour, A. R. Webster, "Ray-tracing and parabolic equation methods in the modeling of a tropospheric microwave link," IEEE Transactions on Antennas and Propagation, vol. 53, no. 11, pp. 37853791, Nov. 2005.

[18] O. Ozgun, G. Apaydin, M. Kuzuoglu, L. Sevgi, "PETOOL: MATLABbased one-way and two-way split-step parabolic equation tool for radiowave propagation over variable terrain", Computer Physics Соттиnications, vol. 182, issue 12, pp. 2638-2654, Dec 2011.

[19] T. E. Bogale and L. B. Le, Beamforming for multiuser massive MIMO systems: Digital versus hybrid analog-digital, in Proc. IEEE Global Telecommun. Conf. (GLOBECOM), Austin, Tx, USA, 1012 Dec. 2014. 